\title{
Supply Chain Management of Antiretroviral Drugs in Public Health Facilities in Eastern Ethiopia
}

\author{
Tesfaye Gabriel ${ }^{1 *}$, Tadesse Bekele Tafesse ${ }^{2}$ \\ ${ }^{1}$ Department of Pharmaceutics and Social Pharmacy, School of Pharmacy, College of Health Sciences, Addis Ababa University, P. O. Box 1176, \\ Addis Ababa, ETHIOPIA. \\ ${ }^{2}$ School of Pharmacy, College of Health and Medical Sciences, Haramaya University, P. O. Box 125, Harar, ETHIOPIA.
}

\begin{abstract}
Objective: Present study is aimed at assessing the supply chain management (SCM) of drugs in selected public health facilities in Dire Dawa City Administration, Harari Region and Jigjiga Zone in Somali Region, Eastern Ethiopia. Methods: A systematic random sampling technique was employed to select the public health facilities from each of the corresponding study areas. Quantitative data were collected using both retrospective and prospective structured observational checklist and structured questionnaire. Data were analyzed using EPI INFO Version 3.5.1 and SPSS version 16.0. Results: A total of 384 HIV/AIDS patients $(47.9 \%$ males and $52.1 \%$ females) were included in the study from three hospitals and seven health centers with mean age of 32.8 years (SD \pm 11.9 years). Ninety percent of the health facilities dispensed the ARV drugs to patients when they came for resupply within 1-3 months. Only thirty percent of the health facilities had received all the ordered quantities of ARV drugs. Zidovudine/ Lamivudine/Nevirapine, Tenofovir/Lamivudine/Efavirenz and Zidovudine/ Lamivudine/Efavirenz combinations were the most commonly prescribed ARV regimens with $31.9 \%, 19.8 \%$ and $15.1 \%$, respectively. Patients who
\end{abstract}

used substances such as khat, cigarette and alcohol along with the ARV drugs were 4.6 times more likely to miss their proper daily dose usage as compared to those who did not use (AOR $=4.619$ [95\% Cl: 2.069-10.313]). Conclusions: The patients should adhere to the right dose at the right time but the health facilities sometimes received not all the quantities of ARV drugs that they have ordered. Zidovudine containing ARV regimen was the most commonly prescribed regimen.

Key words: Adherence, ARV Drugs, Drug Resistance, Supply Chain Management, HIV/AIDS Patients, HAART

\section{Correspondence:}

Tesfaye Gabriel, Department of Pharmaceutics and Social Pharmacy, School of Pharmacy, College of Health Sciences, Addis Ababa University, P. O. Box 1176, Addis Ababa, ETHIOPIA.

Phone: +251912049154

Email: tesfu.gabriel@gmail.com

DOI: 10.5530/jyp.2017.9.109

\section{INTRODUCTION}

The increasing demand of global AIDS treatment poses unprecedented challenges for SCM, since each point of dispensing ARV drugs (i.e., clinic, hospital, and community outreach worker) must have access to a customized and predictable supply of ARVs and other drugs at all times. ${ }^{1}$ However, a major challenge is maintaining continuous supplies of ARV drugs and preventing stock outs. ${ }^{2,3}$ Ensuring an uninterrupted supply of ARV drugs is critical to minimize the emergence of HIV drug resistance, protect the health and well-being of patients, and ultimately reach universal access goals. ${ }^{4}$

The launching of the free ARV treatment initiative in Ethiopia was characterized with a large infusion of commodities which require high logistics capacity. The success of this expanded HIV/AIDS program is dependent on ability to reliably and consistently supply of the essential commodities. $^{5}$

HIV treatment requires an effective supply chain to ensure that ARV drugs are available at all times so as not to cause treatment interruptions. Therefore, ARV SCM must take the following issues into consideration: special distribution requirements for ARVs and diagnostics, constant stock availability, and ARVs are high-value, high-demand products which require extra security. ${ }^{6}$

Uninterrupted supply of quality, safe, effective and comprehensive ARVs for treatment of HIV/AIDS is a pre-requisite and a challenge for ART programs. ARV drugs must travel to treatment sites via a secure and reliable supply chain to ensure the quality, safety and efficacy of formulations. ${ }^{7}$
So far, no work has been reported and documented on SCM of ARV drugs in Eastern Ethiopia and new results and conclusions obtained from this study are recommended for the Federal Ministry of Health and Pharmaceuticals Fund and Supplies Agency of Ethiopia to fill any gaps in the SCM of ARV drugs. Hence, the objective of this study was to assess the SCM of ARV drugs in selected public health facilities in Dire Dawa City Administration, Harari Region and Jijiga Zone in Somali Region, Eastern Ethiopia.

\section{METHODS}

The study was conducted in ten selected public health facilities in Dire Dawa City Administration, Harari Region and Jijiga Zone in Somali Region, Eastern Ethiopia from February 01- May 31, 2014. Health facility based cross-sectional study with quantitative method was employed to assess the SCM in the public health facilities selected purposively. Regarding the evaluation of ART drug use, a single population proportion formula was applied; a total sample size of 384 patients on active ART was included in the study. PLWHA who are in pre-ART and PLWHA who were seriously ill were excluded from the study. An ethical clearance was obtained from Institutional Research Ethics and Review Committee of College of Health and Medical Sciences, Haramaya Univeristy and then delivered to Regional Health Bureaus of Dire Dawa City Administration, Harari Region and Jijiga Zone in Somali Region and the selected public health facilities in the study area. 


\section{RESULTS}

Socio-demographic characteristics of the respondents

A total of three hospitals and six health centers were included in the study and about $74.7 \%$ of respondents were selected from hospitals and the remaining was from health centers. A total of 384 HIV/AIDS patients: $184(47.9 \%)$ males and $200(52.1 \%)$ females were included in the study from the ART units providing pharmacy service with a response rate of $100 \%$. The mean age of the respondents was 32.79 years (SD \pm 11.86 years). The majority of the patients were in the age range of 35-49 years $144(37.5 \%)$ and 25-34 years $126(32.8 \%)$. One hundred ninety five (50.8\%) of the respondents were married, followed by 130 (33.9\%) single respondents. Sixty two (16.1\%) of the patients were illiterate. Most of the respondents, i.e. $236(61.5 \%)$ were unemployed.

\section{Supply of ARV drugs}

Ninety percent of the health facilities dispensed the ARV drugs to patients when they come for resupply within 1-3 months but most of them were supplied within two months while $90 \%$ of the health facilities receive the ARV drugs bimonthly. More than half (60\%) of the health facilities were sometimes received all the quantities of ARV drugs that they have ordered (Table 1).

\section{Storage condition of ARV drugs}

Storage condition of ARV drugs were arranged on shelves with $70 \%$ clear visibility of the identification of product label, expiry dates and manufacturing dates. Only $70 \%$ of ARV products were stored at the appropriate temperature $\left(8-30^{\circ} \mathrm{C}\right)$ including cold chain storage $\left(2-8^{\circ} \mathrm{C}\right)$. On the other hand, only $50 \%$ of the facilities currently had sufficient storage space for existing ARV products \& planned program expansion. Table 2 shows storage condition of ARV drugs in the health facilities.

\section{ARV drug regimens prescribing pattern}

The most commonly prescribed ARV regimens in the facilities were AZT/3TC/NVP (Zidovudine/Lamivudine/Nevirapine) 123 (31.9\%), TDF/3TC/EFV (Tenofovir/ Lamivudine/ Efavirenz) 76 (19.8\%) and 58 (15.1\%) AZT/3TC/EFV (Zidovudine/Lamivudine/Efavirenz) combinations. One hundred eighty one (47.0\%) of people living with HIV/ AIDS took zidovudine containing ARV regimen, which was the most commonly prescribed ARV regimens in the health facilities (Table 3).

Table 1: Supply of ARV drugs

\begin{tabular}{|c|c|c|c|c|c|}
\hline Variables & Monthly & Bimonthly & 3months & Quarterly & No fixed time \\
\hline $\begin{array}{l}\text { How much of a supply is dispensed to } \\
\text { patients when they come for resupply }\end{array}$ & & $9(90 \%)$ & & & $1(10 \%)$ \\
\hline \multirow[t]{2}{*}{ How often do you receive supplies } & & $9(90 \%)$ & & $1(10 \%)$ & \\
\hline & \multicolumn{2}{|c|}{ Always } & Sometimes & \multicolumn{2}{|r|}{ Never } \\
\hline $\begin{array}{c}\text { Do you receive the quantities of ARV } \\
\text { drugs that you ordered }\end{array}$ & \multicolumn{2}{|c|}{$3(30 \%)$} & $6(60 \%)$ & \multicolumn{2}{|r|}{$1(10 \%)$} \\
\hline
\end{tabular}

Table 2: Storage condition of ARV drugs in the health facilities, 2014

\begin{tabular}{llc}
\multicolumn{1}{c}{ Variables } & \multicolumn{2}{c}{ Frequency (\%) } \\
\cline { 2 - 3 } & Yes & No \\
\hline Identification of product label, expiry dates \& manufacturing dates clearly visible & $7(70 \%)$ & $3(30 \%)$ \\
ARV drugs stored \& organized to FEFO procedures & $9(90 \%)$ & $1(10 \%)$ \\
ARV drugs stored in dry, well lit, well-ventilated store room & $8(80 \%)$ & $2(20 \%)$ \\
Products stored at the appropriate temperature $\left(8-30^{\circ} \mathrm{C}\right)$ \& Including cold chain storage $\left(2-8^{\circ} \mathrm{C}\right)$ & $7(70 \%)$ & $3(30 \%)$ \\
Current storage space is sufficient for existing products \& planned program expansion & $5(50 \%)$ & $5(50 \%)$ \\
Fire safety equipment available \& accessible & $5(50 \%)$ & $5(50 \%)$ \\
Products stacked at least $30 \mathrm{~cm}$ away from the walls and other rows & $7(70 \%)$ & $3(30 \%)$ \\
Products stacked no more than $2.5 \mathrm{~m}$ high & $9(90 \%)$ & $1(10 \%)$ \\
Products stacked at least $10 \mathrm{~cm}$ off floor & $8(80 \%)$ & $2(20 \%)$ \\
\hline
\end{tabular}

Table 3: ART regimen prescribed in the health facilities, 2014

\begin{tabular}{ccccc}
\hline ARV Drug Regimens & Frequency & Percent & \multicolumn{2}{c}{ Sex } \\
\cline { 4 - 5 } & & 31.9 & $67(54.5 \%)$ & $56(45.5 \%)$ \\
AZT/3TC/NVP & 123 & 15.1 & $27(46.6 \%)$ & $31(53.4 \%)$ \\
AZT/3TC/EFV & 58 & 19.8 & $43(56.6 \%)$ & $33(43.4 \%)$ \\
TDF/3TC/EFV & 76 & 2.1 & $4(50.0 \%)$ & $4(50.0 \%)$ \\
TDF/3TC/Kaletra & 8 & 14.1 & $29(53.7 \%)$ & $25(46.3 \%)$ \\
TDF/3TC/NVP & 54 & 6.8 & $11(42.3 \%)$ & $15(57.7 \%)$ \\
D4T/3TC/NVP- & 26 & 10.2 & $19(48.7 \%)$ & $20(51.3 \%)$ \\
Other Regimens & 39 & & $200(52.1 \%)$ & $184(47.9 \%)$ \\
\hline
\end{tabular}

Other regimens include AZT/Kaletra, AZT/DDI, TDF/ABC/Lopinavir/r, TDF/DDI/Lopinavir/r 
According to the data obtained in this study, $81.0 \%$ and $12.0 \%$ of people living with HIV were on the standard first-line regimen for adults and pediatrics, respectively while $7.0 \%$ uses second line pediatrics ARV regimens (Figure 1). Forty one percent of the total patients who were receiving ARV drugs had forced at least one case of treatment course change due to different reasons (Figure 2). From drugs that were used for prevention of occurrence of opportunistic infection, $131(34.1 \%)$ of the patients used cotrimoxazole followed by 15 (3.9\%) amoxicillin (Figure 3 ).

\section{Initiation criteria and reports about ARV drugs effects on the patients}

Ninety percent of the health facilities were using laboratory facilities that were used to detect the status of the HIV patients in order to initiate ARV drugs mainly dependent on the CD4+ count and clinical stages. Seventy percent of the health institutions were monitored side effect of ARV drugs but only $20 \%$ of them report to the higher level about the side effect observed. On the other hand, $100 \%$ of the health facilities reported the number of patients on ARV to higher level. With regard of the ARV resistance, $60 \%$ of the health facilities monitor but $40 \%$ of them were reported the cases to higher level. As only the most common Adverse Drug Reactions (ADRs) are detected during pre-marketing trials, it is, therefore, important to devise methods for quick detecting ADRs. This could be carried out by post-marketing surveillance, i.e., ADRs monitoring. Hence, all health professionals have the responsibility to report any unique ADR observed to Food, Medicine and Healthcare Administration Control Authority (FMHACA) of Ethiopia.

\section{Patients' use of ARV drugs}

Out of the 384 patients who received ARV drugs, a total of 50 (13.0\%) patients have missed their proper dose usage and more than half of them were females. The majority of the patients who have missed their dose lie in the age range of 35-49 years that accounts $20(40 \%)$ and 25-34 years $13(26.0 \%)$ from the total patients who have missed dose problems. Out of the 62 patients in the age range of 15-24 have accounted the largest missed dose.

\section{Determinants of missed dose of patients receiving ARV drugs}

Determinants, which were found to be significant by binary regression were introduced to multiple logistic regression. From all covariates studied, illiterate patients were 3.8 times more likely to miss their daily dose than patients who were above secondary (Adjusted Odds Ratio [AOR] $=3.804$ [95\% CI: 1.300-11.133]). Patients who took one drug (fixed combination ART) were 5.32 times more likely to missed their regular dose than patients who took more than one drug per day on average $(\mathrm{AOR}=5.327(1.990-14.257))$.

The odds of missed dose were 3.95 times more likely among patients who have no knowledge about proper dose \& frequency of the ARV drugs usage $(\mathrm{AOR}=3.951$ [95\% CI: 1.475-10.584]) compared to those who have the knowledge. On the other hand, sex, age, marital Status, employment condition and disclosure of patients who receive ARV drugs was not found to affect the utilization of proper daily dose of the patients significantly (Table 4). Majority of the respondents 273 (71.1\%) disclosed their HIV results to at least one person and 111 (28.9\%) were not disclosed themselves from which majority of them were females.

Patients who use substances such as khat, cigarette and alcohol along with the ARV drugs were 4.6 times more likely to miss their proper daily dose usage as compared to those who did not use (AOR $=4.619$ [95\% CI: 2.069-10.313]). The majority of the respondents 314 (81.8\%) did not take any substance; only $70(18.2 \%)$ of the participants take at least one type of substance (smoking cigarette, taking alcohol, or chewing khat). Out

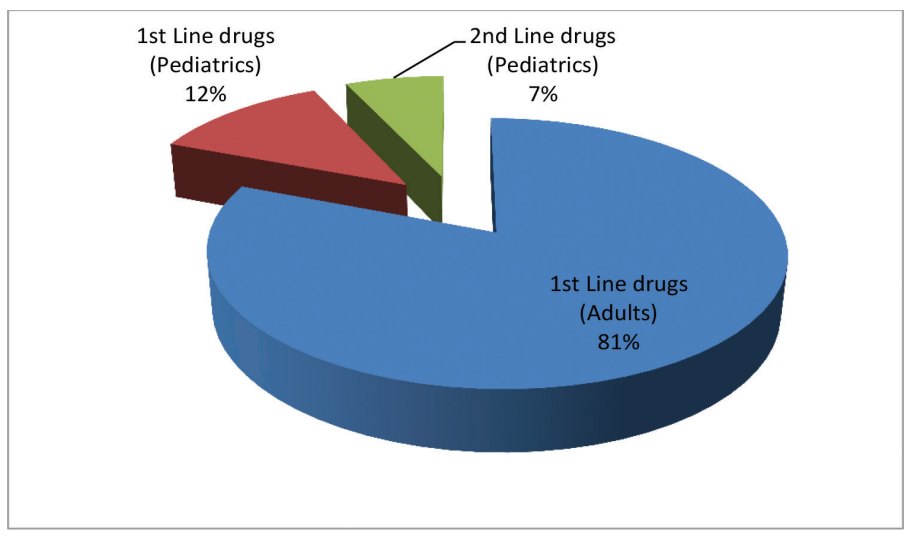

Figure 1: ARV regimens prescribed in terms of first and second line

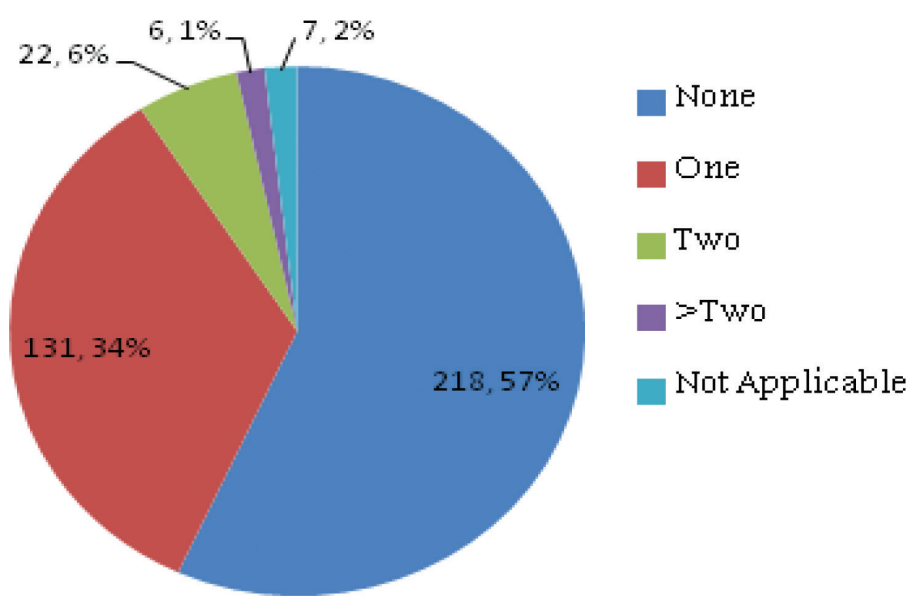

Figure 2: Number of Treatment course changed

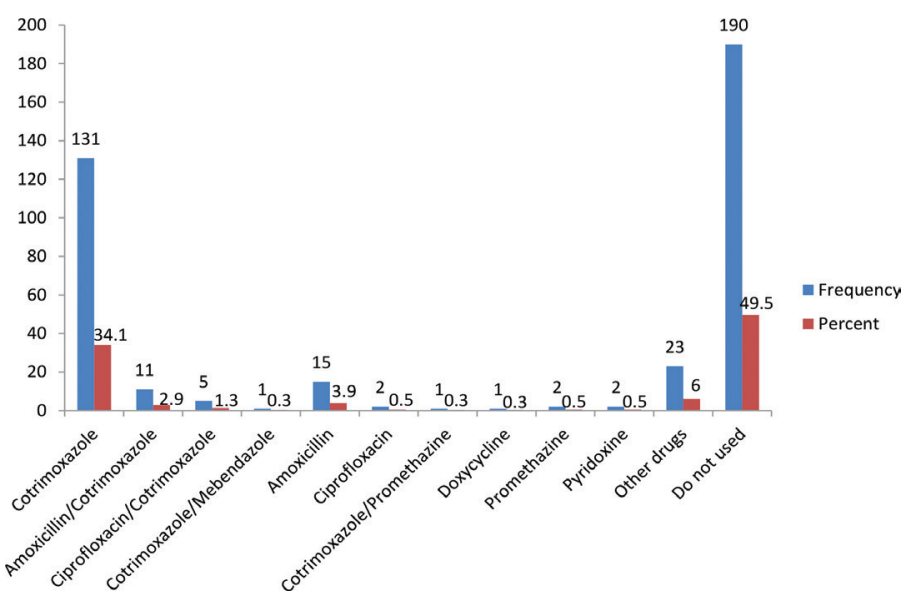

Figure 3: Drugs prescribed for opportunistic infection prevention

of these 14 (3.6\%) of them drunk alcohol and chewed khat, 50 (13.0\%) chewed khat and the others smoke cigarette occasionally. Majority of the respondents $334(87.0 \%)$ did not miss their daily dose.

\section{DISCUSSION}

In Ethiopia, the number of health facilities providing ART services, mainly public facilities, has expanded, enabling the enrolment of hundreds of thousands of PLWHA from time to time as the drugs are provided free of charge. ${ }^{8}$ 
Table 4: Determinants of missed dose of patients receiving ARV drugs, 2014

\begin{tabular}{|c|c|c|c|c|}
\hline \multirow[t]{2}{*}{ Variables } & \multicolumn{2}{|c|}{ Missed Dose } & \multirow[t]{2}{*}{ COR $(95 \% \mathrm{Cl})$} & \multirow[t]{2}{*}{ AOR $(95 \% \mathrm{Cl})$} \\
\hline & Yes $(\mathrm{N}, \%)$ & No $(\mathrm{N}, \%)$ & & \\
\hline \multicolumn{5}{|l|}{ Sex } \\
\hline Male & $22(12.0 \%)$ & $162(88.0 \%)$ & 1 & \\
\hline Female & $28(14.0 \%)$ & $172(86.0 \%)$ & $1.199(0.659-2.180)$ & \\
\hline Total & $50(13.0 \%)$ & $334(87.0 \%)$ & & \\
\hline \multicolumn{5}{|l|}{ Age } \\
\hline$<15$ & $1(4.5 \%)$ & $21(95.5 \%)$ & $0.310(0.032-2.983)$ & \\
\hline $15-24$ & $12(19.4 \%)$ & $50(80.6 \%)$ & $1.560(0.457-5.320)$ & \\
\hline $25-34$ & $13(10.3 \%)$ & $113(89.7 \%)$ & $0.748(0.225-2.480)$ & \\
\hline $35-49$ & $20(13.9 \%)$ & $124(86.1 \%)$ & $1.048(0.331-3.323)$ & \\
\hline$>49$ & $4(13.3 \%)$ & $26(86.7 \%)$ & 1 & \\
\hline \multicolumn{5}{|l|}{ Marital Status } \\
\hline Single & $21(16.2 \%)$ & $109(83.8 \%)$ & $1.070(0.458-2.503)$ & $1.211(0.435-3.370)$ \\
\hline Married & $20(10.3 \%)$ & $175(89.7 \%)$ & $0.635(0.272-1.481)$ & $0.688(0.268-1.768)$ \\
\hline Divorced/Widowed & $9(15.3 \%)$ & $50(84.7 \%)$ & 1 & 1 \\
\hline \multicolumn{5}{|l|}{ Educational Status } \\
\hline Illiterate & $14(22.6 \%)$ & $48(77.4 \%)$ & $2.625(1.085-6.353)^{*}$ & $3.804(1.300-11.133)^{\star}$ \\
\hline Can read and write & $2(6.2 \%)$ & $30(93.8 \%)$ & $0.600(0.124-2.894)$ & $0.711(0.137-3.678)$ \\
\hline Primary (1-8) & $16(15.1 \%)$ & $90(84.9 \%)$ & $1.600(0.689-3.715)$ & $1.589(0.588-4.295)$ \\
\hline Secondary (9-12) & $8(9.5 \%)$ & $76(90.5 \%)$ & $0.947(0.356-2.521)$ & $0.538(0.178-1.629)$ \\
\hline Above Secondary & $10(10.0 \%)$ & $90(90.0 \%)$ & 1 & \\
\hline \multicolumn{5}{|l|}{ Employment Status } \\
\hline Employed & $18(12.2 \%)$ & $130(87.8 \%)$ & 1 & \\
\hline Unemployed & $32(13.6 \%)$ & $204(86.4 \%)$ & $1.133(0.611-2.102)$ & \\
\hline \multicolumn{5}{|l|}{ Substance use } \\
\hline Yes & $16(22.9 \%)$ & $54(77.1 \%)$ & $2.440(1.259-4.729)^{*}$ & $4.619(2.069-10.313)^{\star}$ \\
\hline No & $34(10.8 \%)$ & $280(89.2 \%)$ & 1 & 1 \\
\hline \multicolumn{5}{|c|}{ Number of drugs prescribed } \\
\hline One & $10(32.3 \%)$ & $21(67.7 \%)$ & $3.826(1.642-8.917)^{*}$ & $5.327(1.990-14.257)^{\star}$ \\
\hline Two & $11(12.1 \%)$ & $80(87.9 \%)$ & $1.105(0.528-2.313)$ & $0.948(0.420-2.140)$ \\
\hline${ }^{3}$ Three & $29(11.1 \%)$ & $233(88.9 \%)$ & 1 & 1 \\
\hline \multicolumn{5}{|l|}{ Disclosure } \\
\hline Yes & $30(11.0 \%)$ & $243(89.0 \%)$ & 1 & 1 \\
\hline No & $20(18.0 \%)$ & $91(82.0 \%)$ & $1.780(0.963-3.293)$ & $2.004(0.958-4.190)$ \\
\hline \multicolumn{5}{|c|}{ Knowledge (Dose \& Frequency of drugs) } \\
\hline Yes & $41(11.5 \%)$ & $316(88.5 \%)$ & $3.854(1.625-9.141)^{*}$ & $3.951(1.475-10.584)^{*}$ \\
\hline No & $9(33.3 \%)$ & $18(66.7 \%)$ & 1 & 1 \\
\hline
\end{tabular}

COR-Crude Odds Ratio, AOR-Adjusted Odds Ratio, ${ }^{*}$ statistically significant, CI-Confidence Interval

The most commonly prescribed ARV regimens in the facilities were AZT/3TC/NVP (31.9\%), TDF/3TC/EFV (19.8\%) and AZT/3TC/EFV (15.1\%), followed by TDF/3TC/EFV (19.8\%), TDF/3TC/NVP (14.1\%), D4T/3TC/NVP (6.8\%), TDF/3TC/Kaletra (2.1\%) and other regimens (10.2\%). The result of this study was not consistent with the studies done in Bedele Hospital, Ethiopia, ${ }_{11}^{1}$ Addis Ababa, Ethiopia ${ }^{12}$ and Nekemte Hospital, Ethiopia, ${ }^{13}$ where AZT/3TC/NVP accounts for $15.5 \%, 14 \%$ and $4.2 \%$, respectively. On the other hand, the result of this study was almost consistent with the studies done in Southern India, ${ }^{14}$ South Ethiopia, ${ }^{15}$ Côte d'Ivoire ${ }^{16}$ and Swaziland ${ }^{17}$ where AZT based ARV regimens accounted $49.8 \%, 42.42 \%, 42.0 \%$ and $37.9 \%$, respectively.
Revised WHO guidelines advise changing the eligibility criteria to start ART regimen options for all patients, regardless of pre-ART viral load or CD4 cell count and propose a move away from several Nucleoside Reverse Transcriptase Inhibitors (NRTIs). ${ }^{18}$ However, most of the health facilities were still using those regimens including zidovudine and didanosine as part of an initial ART regimen. In December 2013, Ethiopia adopted the new WHO integrated guidelines for treatment, in which adults with CD4 below 500, all pregnant women and all TB patients independent of CD4 count are eligible for treatment. ${ }^{19}$

Nearly $41.0 \%$ of the total patients was forced at least one treatment course change due to drug associated side effects such as rash, gastrointestinal 
upset, amnesia, etc. Thus, PLWHA were found to use additional drugs to prevent the occurrence of opportunistic infections and 149 (38.8\%) of ART patients were on Cotrimoxazole Preventive Therapy which was much lower as compared to Malawi (94.0\%). ${ }^{20}$

ART adherence levels of $\geq 95 \%$ minimize HIV drug resistance and optimize measures of patient outcomes. ${ }^{21}$ Previous studies in Ethiopia were using only self-reported dose adherence as a measurement. ${ }^{22,23}$ Our data were also based on self-reported dose adherence. The overall ARV drugs dose adherence (no dose missed or delayed for greater than or equal to 90 minutes) among the study respondents was 334 (87.0\%) based on self-report assessment of missed doses (dose adherence) in a one-week recall at baseline with $95 \%$ of prescribed doses. The treatment adherence level found in this study was higher in comparison to studies conducted previously in Botswana (54.0\%) ${ }^{24}$ and Addis Ababa, Ethiopia (81.2\%). ${ }^{22}$

The majority of the respondents $314(81.8 \%)$ did not take any type of substance. The patients being active substance user found in this study were relatively higher in comparison to studies conducted in South Ethiopia $(3.8 \%)^{23}$ but lower than the study conducted in Southwest Ethiopia (7.2\%). ${ }^{25}$ The reasons patients cited for missing doses or delays in dosing time in our study were similar with other studies, which includes being sick, forgetting, work load, lack of transportation for re-supply, and associated side effects..$^{22,23}$

Patients who took one drug (fixed combination ART) per day were 5.32 times more likely to miss their regular dose medication than patients who took more than one drug per day on average (AOR=5.327 (1.990-14.257)). This result was inconsistent to studies done in Eldoret, Kenya $^{26}$ in which adherence decreases as the number of daily taking drugs increases.

Other variables like sex, age, marital status, employment condition and disclosure of patients who receive ARV drugs, which were significant predictors of dose adherence in other studies, did not show association with missed dose of medication in our study. Some results were reported by other study in Eldoret, Kenya ${ }^{26}$ that age, sex, and marital status did not significantly affect the dose adherence rate of the participants.

The current study showed that $90 \%$ of the health facilities dispensed the ARV drugs to patients when they come for resupply within 1-3 months and $90 \%$ of the health facilities receive the ARV drugs bimonthly which was double than the case of Malawi that received the ARV drugs quarterly. ${ }^{3}$ Only $70 \%$ of the ARV products were stored at the appropriate temperature $\left(8-30^{\circ} \mathrm{C}\right)$ including cold chain storage $\left(2-8^{\circ} \mathrm{C}\right)$. An effective and dedicated storage space provides the correct environment for the storage of medicines and commodities and assists the efficient flow of supplies. ${ }^{27}$

Around $90 \%$ of the health facilities followed First Expiry First Out (FEFO) storage protocol. Inadequate storage space contributed for few wrong storage condition with similar situation observed in Sierra Leone..$^{10}$ Additionally, Singh et al and Kant et al reported the ABC and VED matrix analysis systems should be adopted for optimal and rational use of resources and elimination of out-of-stock situations in hospital pharmacy. ${ }^{28,29}$ Kokilam et al suggested the problems associated with stock-outs and wastage can be minimized upon implementation of corrective measures in the areas of procurement, drug quantification, distribution, and inventory control. ${ }^{30}$

\section{CONCLUSION}

In order to maximize the benefit of ARV therapy, patients should adhere to the right dose at the right time as an intervention against barriers to adherence but the health facilities sometimes received not all the quantities of ARV drugs that they have ordered. Zidovudine containing ARV regimen was the most commonly prescribed ARV regimens in the health facilities.
From those HIV patients who have missed their proper daily dose and frequency of ARV drugs, females were the dominant with the age range of 35-49 years. Most of the health facilities were monitoring side effect of ARV drugs but only few of them reported the observed side effect to the higher level. Only half of the health facilities currently had sufficient storage space for existing ARV products \& planned program expansion for proper storage condition of ARV drugs.

\section{ACKNOWLEDGEMENT}

The authors are grateful to Haramaya University and Clinton Health Access Initiative (CHAI) for their financial support. We would also like to extend our gratitude to physicians, pharmacists and nurses working at different health facilities for their kind assistance during data collection.

\section{COMPETING INTEREST}

The authors declare that they have no competing interests.

\section{AUTHORS' CONTRIBUTION}

TB and TG involved in conception of the original idea, helped to draft the proposal, participated in all implementation stages of the project, and write up; both reviewed it critically and involved in all implementation stages of the project and TB conducted the write up. Both authors read and approved the final version of the manuscript.

\section{REFERENCES}

1. Xiong W, Hupert N, Hollingsworth EB, O'Brien ME, Fast J, Rodriguez WR. Can modeling of HIV treatment processes improve outcomes? Capitalizing on an operations research approach to the global pandemic. BMC Health Serv Res. 2008;8:166 doi: 10.1186/1472-6963-8-166. http://www.biomedcentral. com/1472-6963/8/166. Date accessed on 28/08/2012.

2. Decroo T, Telfer B, Biot M, Maike're J, Dezembro S, Cumba LI, et al. Distribution of ARV Treatment through Self-Forming Groups of Patients in Tete Province, Mozambique. J Acquir Immune Defic Syndr. 2011;56:39-44.

3. Schouten EJ, Jahn A, Ben-Smith A, Makombe SD, Harries AD, Aboagye-Nyame $F$, et al. ARV drug supply challenges in the era of scaling up ART in Malawi. J Int AIDS Soc. 2011;14:S4. doi: 10.1186/1758-2652-14-S1-S4

4. WHO, UNAIDs, and UNICEF. Towards Universal Access: Scaling up priority HIV/ AIDS interventions in the health sector. Progress Report. Geneva, World Health Organization, 2010.

5. Alemayehu L. Assessment of supply management current status for ARV therapy (ART) in Oromia national regional state, Ethiopia. Ethiopian Pharmaceutical Association Annual Conference Abstract, 2009.

6. Mubangizi DB, James BJ, Ogbuabo M. AIDS Relief, Supply Chain Manual, Catholic Relief Services, 2011.

7. PEPFAR, The United States President's Emergency Plan for AIDS Relief. Bringing Hope: Supplying ARV Drugs for HIVIAIDS Treatment. Report on ARV Drugs for HIV/AIDS Treatment. Report to Congress Mandated by H.R. 3057, 2006.

8. FMOH (Federal Democratic Republic of Ethiopia Ministry of Health). (October 2010). Health Sector Development Programme IV 2010/11 - 2014/15

9. Subhash CA. Adequate quality of HIV drugs must be ensured. BMJ. 2002;324:235-6. 2002; 324.

10. USAID: DELIVER PROJECT. Supply Chain Assessment for ARV Drugs and HIV Test Kits. National HIV/AIDS Secretariat/Ministry of Health and Sanitation, 2007.

11. Mekonnen Y, Molla G. Reason for regimen change among HIV patients on initial highly active ARV therapy in Bedele hospital ART clinic, Ethiopia. J Biotechnol Biosafety. 2014;2:116-22.

12. Jima YT, Angamo MT, Wabe N. Causes for ARV regimen change among HIV/ AIDS patients in Addis Ababa, Ethiopia. Tanzan J Health Res. 2013;15:1.

13. Wube M, Tesfaye A, Hawaze S. ARV Therapy Regimen Change among HIV/ AIDS Patients in Nekemt Hospital: A Primary Care Hospital in Oromia Regional State, Ethiopia. J Appl Pharm Sci . 2013;3(08):36-40.

14. Kumarasamy N, Venkatesh KK, Devaleenol B, Saghayam S, Manohar D, Poongulali $\mathrm{S}$, et al. Safe substitution to zidovudine among HIV-infected patients initiated on stavudine-containing highly active ARV therapy from a resource-limited setting. Int J Infect Dis. 2009;13:e360-e4.

15. Woldmedhin B, Wabe NT. The Reason for Regimen Change among HIV/AIDS Patients Initiated on First Line Highly Active ARV Therapy in Southern Ethiopia. N A J Med Sci. 2012;4:19-23.

16. Messou E, Anglaret X, Duvignac J, Konan-N'Dri E, Gnokoro J, Karcher S, Tanoh A, N'Dri-Yoman T, Seyler C. ARV treatment changes in adults from Côte d'Ivoire: 
the roles of tuberculosis and pregnancy. AIDS. 2010;24:93-9.

17. Takuva S, Louwagie G, Zuma K, Okello V. Durability of First Line ARV Therapy: Reasons and Predictive Factors for Modifications in a Swaziland Cohort. J Antivir Antiretrovir. 2012; 4:1

18. WHO (World Health Organization). Consolidated guidelines on HIV prevention, diagnosis, treatment and care for key populations, 2014.

19. FDRE (Federal Democratic Republic of Ethiopia). Country Progress Report on the HIV Response, 2014.

20. Malawi Government. 2012 global AIDS response progress report: Malawi country report for 2010 and 2011, 2012.

21. Carlucci JG, Kamanga A, Sheneberger R, Shepherd BE, Jenkins CA, Spurrier J, et al. Predictors of adherence to ARV therapy in rural Zambia. J Acquir Immune Defic Syndr. 2008;47:615-22.

22. Tadios Y, Davey G. ARV treatment adherence and its correlates among people living with HIV/AIDS on highly active ARV therapy in Addis Ababa, Ethiopia. Ethiop Med J. 2006;44:237-44.

23. Markos E, Worku A, Davey G. Adherence to ART in PLWHA at Yirgalem Hospital, South Ethiopia. Ethiop J Health Dev. 2008;22(2):174-9.

24. Weiser S, Wolfe W, Bangsberg D, Thior I, Gilbert P. Makhema J, et al. Barriers to ARV adherence for patients living with HIV infection and AIDS in Botswana.
J Acquir Immune Defic Syndr. 2003;34:281-8.

25. Tiyou A, BelachewT, Alemseged F Biadgilign S. Predictors of adherence to ARV therapy among people living with HIV/AIDS in resource limited setting of southwest Ethiopia. AIDS Res Ther. 2010;7:39.

26. Talam NC, Gatongi PJ, Rotich J, Kimaiyo S. Factors affecting ARV drug adherence among hiv/aids adult patients attending HIV/AIDS clinic at Moi teaching and referral hospital, Eldoret, Kenya. East Afr J Public Health. 2008:5(2):74-8.

27. Iqbal MJ, Geer MI, Dar PA. Medicines Management in Hospitals: A Supply Chain Perspective, Sys Rev Pharm. 2017:8:80-5.

28. Singh S, Gupta AK, Latika, Devnani M. ABC and VED Analysis of the Pharmacy Store of a Tertiary Care, Academic Institute of the Northern India to Identify the Categories of Drugs Needing Strict Management Control. J Young Pharm. 2015;7:76-80. DOI: 10.5530/jyp.2015.2.4

29. Kant S, Haldar P, Singh A, Kankaria A. Inventory Management of Drugs at a Secondary Level Hospital Associated with Ballabgarh HDSS- An Experience from North India. J Young Pharm. 2015;7:113-7. DOI: 10.5530/jyp.2015.2.9.

30. Kokilam MB, Joshi HG, Kamath VG. Assessment of Pharmaceutical Store and Inventory Management in Rural Public Health Facilities-A study with reference to Udupi District, Karnataka. Pharm Methods. 2015;6(2):53-9. DOI: 10.5530/ jyp.2015.6.7.

Article History: Submission Date : 25-02-2017 ; Revised Date : 30-05-2017; Acceptance Date : 11-06-2017.

Cite this article: Gabriel T and Tafesse TB. Supply Chain Management of Antiretroviral Drugs In Public Health Facilities In Eastern Ethiopia. J Young Pharm. 2017;9(4):571-6 